\title{
Curriculum goals for the training of osteopathic family practice residents for the year 2000
}

\author{
JOEL DICKERMAN, DO \\ PAMELA RICH
}

The osteopathic family practitioner of the future will play a larger role in the delivery of healthcare under managed care. To prepare for this role, osteopathic family practice residents must develop skills in direct medical care, medical education, and healthcare administration. Likewise, these residents need to become familiar with the clinical setting in which these skills are needed, specifically, ambulatory, rural, hospital, and minor emergency care environments.

We propose the development of a curriculum designed to promote these skills and clinical experiences. This curriculum would expand family practice experiences; replace the current required rotations in specialty medicine with longitudinal rotations designed to develop specific family practice skills; include block rotations in hospital care, minor emergency care, and rural medicine; develop model case assignments to teach residents to integrate their roles as provider, educator, and administrator; and entail clinical experiences in medical administration and education. An evaluation process that reflects the goals of the family practice curriculum is key to the success of such a program.

(Key words: Osteopathic family practice curriculum, osteopathic medical education, resident evaluation, American College of Osteopathic Family Physicians, healthcare reform)

Healthcare reform, rising medical costs, and the need to manage ever-growing medical knowledge and technology have placed a demand for an increase

Dr Dickerman is the program director for the Colorado Springs (Colo) Osteopathic Foundation Family Practice Residency program. Ms Rich is the program coordinator for the Colorado Springs (Colo) Osteopathic Foundation Family Practice Residency Program.

Correspondence to Joel Dickerman, Colorado Springs Osteopathic Foundation \& Family Medicine Center, 15 W Cimarron, Colorado Springs, CO 80903.

in the number of family practice physicians. Although osteopathic medicine has a tradition of producing family practice physicians, it will not be enough for us to simply train more students to become osteopathic family physicians. We must also train them to make healthcare more cost-effective, to operate in an ambulatory-care setting, and to engage in primary care research. ${ }^{1}$ To accomplish these goals, osteopathic family practice training programs must develop a curriculum that reflects the emerging role of the family practitioner in healthcare delivery.

Among those factors that shape the training of the osteopathic family practitioner are the role of this physician in healthcare delivery and the practice setting of family practitioners in the future. The role of the family practitioner represents three major functions: provider, educator, and administrator. As a provider, the physician evaluates patient problems, directs medical intervention, and promotes preventive care. As an educator, the physician must keep current with medical knowledge and share this information with colleagues and patients. As an administrator, the physician must assure quality care in a cost-effective manner. Opportunities for family practice physicians can be found in four settings: ambulatory/urban, rural, minor emergency care, and hospital-based medicine.

These roles and settings have emerged as a result of pressures to contain medical costs; the rapid growth of medical technology and knowledge; and the expansion of medical specialties. ${ }^{2,3}$ Rather than making the family physician obsolete, these forces have actually placed this physician at the heart of the public's demand to concentrate on the care of the family unit. What follows is a discussion on how the osteopathic medical profession needs to reshape our family practice training programs to meet these demands.

\section{Roles of the family physician Provider}

In this age of cost containment and "gatekeepers," we must not lose sight of the primary role of the 
osteopathic family physician, which is to provide medical care. This care entails differentiating between problems, using medical modes of therapy, and providing patients with preventive care. Problem differentiation is the process of evaluating poorly defined patient complaints and developing a logical treatment plan. ${ }^{4}$ Family physicians are most often the first medical provider whom patients encounter when they have a medical problem. These complaints are often vague or cannot readily be attributed to a specific disease process. Tools available to the family physician for this evaluation include taking the patient's history and conducting the physical examination; using in-office diagnostic tests; interpreting results from tests conducted in outside laboratories; using consulting services; and evaluating the patient in a longitudinal manner.

Medical therapeutics encompass direct medical care of the patient by the physician. The osteopathic family physician can provide such care through the use of medications; management of chronic disease; the application of osteopathic manipulative treatment; and patient education. Each of these options requires a thorough understanding of the indications and contraindications for treatment and a basic level of expertise in each of the treatment options available.

Preventive care has evolved beyond simply providing childhood immunizations and performing annual physical examinations. It now encompasses cancer screenings, risk stratification (for example, the evaluation of risk factors contributing to the onset of heart disease), and patient education. Family physicians therefore must foster skills in diagnostic procedures (sigmoidoscopy, colposcopy); and patient communication, both spoken and written words, as well as the ability to use ancillary services.

To help the physician-in-training become proficient in these roles, training programs must have a family practice curriculum that addresses the individual skills needed to be effective in each of these areas. We need to provide students with experiences that develop skills in family practice, rather than promote a resident to become a cardiologist, or a general surgeon for a month. Specialty rotations need to teach those skills necessary to evaluate chest pain or acute abdominal pain as well as to learn to interpret electrocardiograms and abdominal x-ray films. Family physicians must know when they can provide care and when they need to refer patients for care. To that end, we must develop reading lists, lecture schedules, and clinical experiences (such as colposcopy and vasectomy procedures) that foster these universal skills. In this way, the physician can treat all members of the family, regardless of gender or age. ${ }^{5}$

\section{Educator}

The explosion of medical information over the past few decades-and the need to integrate and disseminate this information into the practice of medicine-has prompted the physician to assume the role of educator as well. ${ }^{6}$ Family physicians also are playing an increasing role in primary care research ${ }^{7,8}$ as we seek to understand what impact medical advancements have on patient care.

In this role as educators, physicians need to become proficient in self-education, patient education, and peer education. Self-education is not needed merely to meet requirements of continuing medical education for continued licensure. Rather, it is necessary to provide optimal care for patients. ${ }^{9}$ Use of medical resources (periodicals, lectures, computer programs), case discussions with peers, and goal-setting for patient care all fall under the realm of self-education.

Patient-Patient education includes having direct communication with patients, conducting community lectures, and using ancillary educational services (such as diabetes education programs). Improving communication skills-both spoken and written-lies at the center of patient education, as does the ability to investigate new teaching modalities (videotapes, interactive computer programs).

Peer-Lecture presentation, publication of medical articles, research in primary care, and presentation of patient cases represent some forms of peer education. These types provide an efficient source of medical education, as they allow presenters to gather information from various sources, thereby disseminating the most current medical knowledge.

For family physicians to become effective educators, we must create curriculum goals that emphasize the role of educator as vital to being an effective medical provider. Family physicians in training must be made aware of how integral teaching others is to the optimal care of patients. Educational objectives must be integrated into the everyday clinical experiences of patient care, specifically during patient visits and case presentations. Similarly, research in primary care presented at conferences can be used to improve patient care as well.

\section{Administrator}

As administrators, family physicians need to optimize communication and to ensure the deliverance of quality, cost-efficient care. Such care encompasses the use of office policies and protocols that maximize resources. Cultivating management skills that include completing paperwork on time 
and staffing the office efficiently prove essential in this role.

To prevent duplication of services and to ensure appropriate care, provider-to-provider communication encompasses clear, concise chart keeping; articulate interfacing with office staff; and thorough case presentations to consulting physicians. Inaccuracies in communicated information can delay patient care and can lead to potential complications if medication dosages or diagnostic test results, for example, are misinterpreted by other physicians.

The process of making sure diagnostic tests and medical treatments are undertaken in a correct manner so that results are accurate, reflecting the patient's actual medical state, falls under quality assurance. This accuracy depends, in part, on being familiar with laboratory tests, $\mathrm{x}$-ray films, and in-office diagnostic tests (electrocardiograms and pulmonary function tests, for example). Treatment modalities, such as cryotherapy, electrocautery, and physical therapy also must be understood well, particularly regarding means of delivery and effectiveness for the desired patient response.

Again, these skills must be taught in the context of direct patient care. Likewise, administrative duties should be addressed while on medical rotations, during medical lectures, and at resident staff evaluations. The goal of the family practice curriculum should not be to groom students to become medical administrators, but rather to incorporate administrative skills into the everyday practice of family medicine.

\section{Family practice settings defined Ambulatory/urban}

Family practice medicine has become increasingly ambulatory-based because of the demand for office-based care. Along with this demand comes an influx of in-office diagnostic equipment and changes in the reimbursement structure. All these changes have made such demands on in-office hours that many physicians have given up or significantly limited their hospital care, surgical assistance, and work in obstetrics. As a result of these factors, long-term, in-depth care has become the cornerstone of family practice medicine. Again, the same communication skills already outlined herein apply in this setting as well.

More than medicine, family practice entails working with home care agencies, supervising inhouse diagnostic testing, and managing patient files and other information. Exposure to family practice medicine clinics as well as in-office settings during rotations affords excellent learning opportunities.

\section{Rural}

Rural medicine cannot simply be considered a return to the "good old country doctor." Physicians working in this setting are often asked to cover in the emergency room as well as to undertake community administrative duties (hospital medical director or supervisor of emergency services, for example). Rural physicians must also be adept at transferring patients to tertiary medical centers when the need arises. Because of the shortage of family physicians in this environment, such residents must not only be exposed to rural medicine, but they also must be taught to be proficient in these necessary skills so that they can feel comfortable in meeting the needs inherent in this setting.

\section{Minor emergency}

The recognition that immediate care can alter the outcome of emergent medical problems and the demand for extended office-based hours has created a burgeoning of minor emergency care centers. With their familiarity with diverse patient populations and medical problems, family physicians are often sought to staff such centers. Therefore, these residents should be exposed to such practice opportunities, including working in fast-track services in community emergency rooms and specialty minor illness centers (after-hours pediatric clinics, for example). More than simply familiarizing themselves with the operations of a minor emergency center, residents should know the special populations served by these centers, whether the patients consist of "weekend warriors" found in sports medicine clinics, or white- (or blue-) collar workers injured on the job and seeking treatment at an occupational medicine center.

\section{Hospital}

Management of hospital patients continues to be a part of urban and rural family practice. Yet, a growing demand exists for family practitioners who specialize in the management of hospitalized patients. These physicians provide family care at any time of the day or night and are readily available to discuss patient care with specialists and ancillary care services. Family practice residents should receive ample exposure to hospital-based family medicine to evaluate this track as a potential practice opportunity. Residents expressing an interest in hospital medicine should pursue inpatient pediatrics and intensive care, as management of such patients after graduation often depends on having documented training in these areas. ${ }^{10}$

\section{Future curriculum}

The American College of Osteopathic Family Physicians (ACOFP) has begun to establish the basic curriculum format needed to develop the Family Practitioner for the Year 2000. The Model Program for Two-Year Basic Standards for Residen- 
cy Training in General Practice has established the importance of ambulatory training for this physician. This standard discusses the need for family practitioners to integrate an ambulatory-based practice with hospital care along with care provided by specialists.

The ACOFP competency-based evaluation program supports the development and maintenance of specific family practice skills. Family practice residents are not simply exposed to clinical situations, but they must possess an understanding of clinical presentations and procedures. Again, the ACOFP document details specific resident skills and a means of evaluating resident competency. Among those skills outlined in this document are the management of patients with heart failure and pediatric patients with acute asthma.

We propose the following curriculum goals to further enhance the aforementioned skills:

- Base the family practice residency on primary care experiences. Expand this clinical training to $3 \frac{1}{2}$ days per week, with $1 / 2$ day dedicated to education (attending lectures, keeping patient charts), and 1 day devoted to longitudinal rotations. Residents would be encouraged to care for a large family practice patient population that will provide them the means to learn speciality care in the context of general medicine. Instead of following up the patient population of one specialist, residents would be encouraged to learn specialty care from that which is provided to the resident's patient population. As such, residents would also discuss their patients' cases with specialists as well as arrange to be with their patients when they are seeing the specialist.

Specialty rotations, then, would consist of longitudinal 1-day-per-week experiences in which learning would center around the resident's own patient population. In addition, residents would provide hospital care and coordinate specialty care for their patients. In this way, residents will learn to integrate various care into a comprehensive practice of family medicine.

- Eliminate block rotations in specialty care. Longitudinal rotations in specialty care would be designed to foster skills family practitioners require. These skills include weekly exposure to cardiac medicine so that residents learn to interpret electrocardiograms and to evaluate patients with chest pain. Rotations in orthopedics would expose residents to office-based care, specifically in casting, evaluating patients, and performing other in-office procedures, such as intra-articular injections. Residents would also be taught when to refer patients to specialists, how to evaluate patients before such referral, and how to discuss their patients' cases clearly with consulting physicians.
Establish block rotations in family practice clin$i c s$. We propose the development of block rotations in minor emergency care, hospital care (including intensive care units, and pediatric medicine), and rural medicine. Such rotations would provide residents with concentrated exposure to various work environments. Residents could then arrange longitudinal rotations in any one of these settings should they desire to pursue a particular career path.

- Assign patient cases. Such a program would entail residents' being responsible for patients with specific disease entities (hypertension, diabetes, for example) as a basis for self-directed learning. Besides being familiar with each patient's complaints, residents would be required to base their research on that patient's disease or medical condition. By searching the literature and speaking with specialists, residents can begin to establish practice guidelines for treating patients with the same medical condition. Assignments would be made on a monthly or quarterly basis. Residents would learn their roles of provider, educator, and administrator in this way. Specifically, residents would care for their patients; discuss that care with consulting physicians; educate their colleagues on the resident's research; and perform administrative duties (such as keeping charts, summarizing cases, and completing forms).

- Expose residents to administrative duties. Such duties should be taught in a manner that enhances patient care. Administrative duties should not be considered to be nuisances. Senior residents could design and staff a mock office based on the level of care they have achieved among their patient population. Junior residents could be required to conduct educational seminars, covering such topics as smoking cessation or parenting. These seminars would be conducted on a monthly basis as part of the resident's assignment. Lectures would be designed to show residents how such seminars improve patient care.

\section{Program evaluation}

Central to the success of any family practice program, the resident evaluation process reflects the goals and objectives of the core curriculum. The competency-based evaluation system developed by the ACOFP supports many of the aforementioned goals. This evaluation process emphasizes fostering specific family practice skills and encourages achievement of a certain proficiency level before residents are graduated from the program. Clinical knowledge, professional judgment, teaching and administrative skills, and overall responsible behavior are at the core of this curriculum and the evaluation process as well. 


\section{Summary Evaluation of Resident}

\section{Resident}

Rotation

Rate on a scale of 1 to 5 ( 5 being highest proficiency).

Preceptor

The Physician as Provider

1. Knowledge of basic sciences

Knowledge of medicine, physiology, pathology.

Knowledge of common disease states

2. Understanding of clinical medicine

Ability to manage chronic illness

Ability to provide preventive care

3. Diagnostic judgment

Ability to order, interpret diagnostic tests

4. Comprehensiveness of treatment plan

Ability to devise treatment plan

Ability to manage the case

5. Understanding of clinical procedures Recognition of patient-care protocols

6. Technical ability

Ability to perform diagnostic procedures

Ability to perform surgical procedures

7. Critical decision making

Ability to recognize, treat medical emergencies

8. Application of osteopathic medicine principles

9. Use of behavior skills, concepts

\section{The Physician as Educator}

10. Interest in self-directed study

Ability to use medical resources (computer, library)

11. Interaction with patient, family

Ability to communicate with patient and

family members (giving instructions)

12. Response to constructive criticism

13. Quality of scientific research

Journal club presentations, participation

\section{The Physician as Administrator}

14. Dedication to medicine

15. Ethical standards

16. Acceptance of administrative tasks

Ability to develop office policy

Ability to supervise, evaluate employees

Ability to determine office staffing, equipment needs

Ability to manage financial matters

Ability to manage time efficiently

17. Interaction with other physicians

18. Interaction with other healthcare professionals

19. Professional demeanor

20. Leadership capabilities

21. Chart-keeping thoroughness

*Resident's signature

Preceptor's signature

Program Director's signature

* (Signature of resident acknowledges receipt and review of evaluation, not necessarily agreement with content.)

Figure. Quarterly Summary Evaluation of Residents, adapted from the American College of Osteopathic Family Physicians. 
We have adapted the ACOFP annual evaluation form to reflect the general goals of the physician as being provider, educator, and administrator (Figure). This form would be used on a quarterly basis to provide resident feedback and to set clinical goals for the next quarter.

\section{Comment}

Family physicians play a vital role in the current delivery of healthcare, particularly in this age of managed healthcare. We must ensure that the osteopathic family practice physicians of the future can fill this multifaceted role and not simply perform as "gatekeepers." To that end, we have proposed a family practice training program designed to expand opportunities and to foster a sense of competency in a variety of settings (urban, rural, and minor emergency care), and roles (provider, educator, and administrator) that are inherent in family practice medicine. To gauge the effectiveness of these curriculum changes, we have also provided a resident's evaluation form. In this way, the curriculum can be adapted to train the most effective osteopathic family physicians possible.

\section{References}

1. Schwenk TL: Family medicine. JAMA 1992;268:356-358.

2. Geyman JP: Training primary care physicians for the 21 st century. Alternative scenarios for competitive vs generic approaches. JAMA 1986 255:2231-2635.

3. Schwerger JE: The second revolution in family practice has begun. Am Fam Physician 1993;48:1238-1240.

4. Rakel RE: Textbook of Family Medicine, ed 4. Philadelphia, $\mathrm{Pa}$, WB Saunders Co, 1990, pp 402-403.

5. Geyman JP: Family medicine as an academic discipline: Progress, challenges, and opportunities. J Fam Pract Med 1990;31:297-303.

6. Evans PR: The changing scene in general practice in Europe. BMJ 1994;308:645-648.

7. Morrell DC: Role of research in development of organization and structure of general practice. BMJ 1991;302:13131316.

8. Lawrence M: Caring for the future. BMJ 1992;305:400-402. 9. Shapiro J: The maturing of family medicine: Challenges to the behavioral sciences. J Fam Pract Med 1988;26:583-584. 10. Council on Long Range Planning and Development, American Medical Association: The future of family medicine. JAMA 1988;260:1272-1279. 